\title{
EDENTULISMO Y NECESIDAD DE TRATAMIENTO PROTÉSICO EN PACIENTES ADULTOS DE LA UNIDAD ACADÉMICA DE SALUD Y BIENESTAR - UCACUE 2016
}

\section{EDENTULISM AND THE NEED FOR PROTETIC TREATMENT IN ADULT PATIENTS OF THE ACADEMIC HEALTH AND WELFARE UNIT - UCACUE 2016}

\author{
Matute-Bueno Xavier $^{1 *}$, Pacheco-Arce María Gabriela ${ }^{2}$ \\ ${ }^{1}$ Catedrático de la Carrera de Odontología de la Universidad Católica de Cuenca. Ecuador \\ 2 Odontóloga Rural del Ministerio de Salud Pùblica de Ecuador. Ecuador \\ *sematute@hotmail.com
}

\begin{abstract}
Resumen
OBJETIVO: Determinar la Frecuencia de Edentulismo y la necesidad de tratamiento protésico en pacientes adultos entre 18 a 70 años de edad de la clínica integral de la Carrera de Odontología en la Unidad Académica de Salud y Bienestar, Cuenca - Ecuador, 2016. MATERIALES Y MÉTODOS: Se realizó un estudio de tipo descriptivo, retrospectivo. La muestra estuvo integrada por 100 adultos de 18 a 70 años de edad, quienes fueron observados clínicamente y evaluados mediante el informe de la Organización Mundial de la Salud (OMS) para personas edéntulas. RESULTADOS:El $100 \%$ de la población presentó edentulismo parcial, dentro de este $87 \%$ edentulismo parcial bimaxilar y $13 \%$ edentulismo parcial unimaxilar de este último se identificó específicamente un $8 \%$ de edentulismo parcial superior y $5 \%$ de edentulismo parcial inferior. CONCLUSIÓN: Se determinó que existe una necesidad de tratamiento protésico parcial inferior del $82 \%$, tratamiento protésico total del $1 \%$ y el $17 \%$ de la población no necesitan un tratamiento prótesico.
\end{abstract}

Palabras clave: Arcada edéntula, Dentadura Completa, Dentadura Parcial.

\begin{abstract}
OBJETIVE: To determine the frecuency of Edentulism and the need for prosthetic treatment in adult patients between aged from 18 to 70 years in comprehensive clinic Race Dentistry at the Academic Unit of Health and Welfare, Cuenca - Ecuador, 2016. MATERIALS AND METHODS:The study was retrospective descriptive. The population was of 100 adults aged 18 to 70 years old who were clinically observed and evaluated by Form by the Organization World Health (WHO). RESULTS:It was determined that $100 \%$ of the population studied presented partial edentulism, with a higher frequency of bimaxilar partial edentulism with $87 \%$ and partial upper edentulism $8 \%$ and partial lower $5 \%$. The highest frequency of partial bimaxillary edentulism was obtained in all ages from 18 to 75 years with a percentage of $87 \%$, a higher frequency was also seen in lower and upper partial edentulism in people under 30 years of age with 27Bimaxilar partial edentulism according to sex has manifested a higher frequency in males with 93\%, in lower partial edentulism the higher frequency is in the female sex with $10 \%$ and a lower frequency of upper partial edentulism in the Male sex with $2 \%$. It was determined that there is a great need for partial inferior prosthetic treatment with $82 \%$, total $1 \%$ and $17 \%$ no need for a treatment. The need for inferior prosthetic treatment is most often seen in the need for partial treatment with $91 \%$, total $1 \%$ and no treatment is required with $8 \%$. CONCLUSION: The total population evaluated was composed of 100 adult patients presented with 100\% partial edentulism, in the way that $87 \%$ represents bimaxillary edentulism and $13 \%$ that comprises upper and lower partial edentulism. Seen in $87 \%$ of the population studied, total $1 \%$ and do not require prosthetic treatment $13 \%$.
\end{abstract}

Key words: Edentulous Jaw, Denture Complete, Denture, Partial.

\section{INTRODUCCIÓN}

La salud bucal no solo se ve relacionada con la presencia de piezas dentarias de forma natural o artificial sino que también se ve relacionada con el estado general del sistema estomatognático, incluyendo las estructuras de soporte, músculos, maxilares y Articulación Temporomandibular, que 
al encontrase en un buen estado lo podríamos definir como salud bucal netamente integral. ${ }^{1}$

Los adultos en general restan importancia en la conservación de la salud bucal observando un aumento en la perdida de piezas dentarias, es decir que estos pacientes pueden presentar edentulismo ya sea parcial o total.

El edentulismo es considerado como un problema irreversible que puede conducir a un deterioro físico, mental, funcional y psicológico ${ }^{2}$ muy frecuente en los Adultos Jóvenes, Medios y Mayores que comprenden edades entre 18 a 100 años de edad. Pueden ser causados por diversos factores, uno de los principales es la caries dental que se define como la destrucción de los tejidos dentarios causado por bacterias de la placa depositada en las superficies dentales. ${ }^{3-8}$ Por lo tanto, la pérdida de dientes se torna un fuerte indicador de la salud en los pacientes adultos y ancianos. En ciertos estudios hechos en Brasil ${ }^{9}$ y en otras partes del mundo señalan la importancia de los conocimientos epidemiológicos acerca de la pérdida de las piezas dentarias, su impacto en la salud pública, la calidad de vida en adultos y ancianos, sus factores socioeconómicos y el ingreso a servicios dentales.

Los estudios confirman que la salud bucodental tiene un gran impacto en la calidad de vida de los pacientes, de manera que si se encuentra afectada por la pérdida de las piezas dentarias estas van a interferir con la pronunciación de las palabras, la alimentación y la vida social del paciente, alterando su calidad de vida. ${ }^{10}$

\section{MATERIALES Y MÉTODOS}

El diseño de la investigación fue descriptivo retrospectivo, en donde se evaluó a 100 pacientes adultos en edades de 18 a 70 años de edad en la Clínica Integral de la Carrera de Odontología, Cuenca-Ecuador 2016.

Los Criterios de Inclusión fueron: adultos entre 18 años en adelante, y los Criterios de exclusión fueron: los pacientes que tenían menos de 18 años y los adultos que presentaban trastornos psicológicos.

Los pacientes involucrados en el estudio aceptaron la participación firmando el consentimiento, de manera que se recolectaron los datos generales y clínicos mediante un formulario utilizado de la OMS (Organización Mundial de la Salud) pudimos evaluar la salud bucal del paciente, su situación y necesidad de tratamiento protésico.

Para el análisis estadístico, la ejecución de tabulaciones y gráficos se utilizó el programa de Microsoft Excel.

\section{RESULTADOS}

Se estudiaron a 100 pacientes de la Clínica Integral de la Universidad Católica de Cuenca de manera que 58 eran mujeres y 42 hombres con un rango de edad entre 18 años a 75 años.
Se encontró el $100 \%$ de los pacientes estudiados que presentaban edentulismo parcial y total, obteniendo resultados de una mayor frecuencia el edentulismo Bimaxilar con un $87 \%$, edentulismo parcial inferior $8 \%$ y una menor frecuencia es parcial superior $5 \%$.

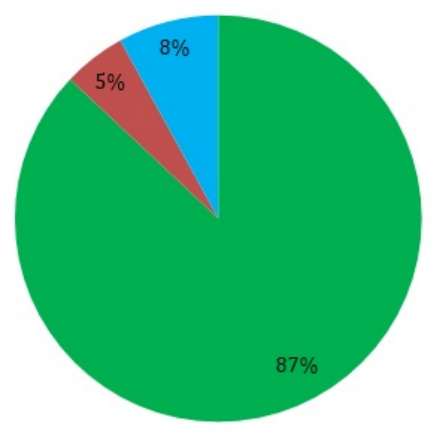

- PARCIAL BIMAXILAR - PARCIAL SUPERIOR - PARCIAL INFERIOR

Grf. 1. Frecuencia de Edentulismo en los pacientes adultos de la Clínica Integral de la Carrera de Odontología en la Unidad Académica de salud y Bienestar 2016.

\begin{tabular}{lcccc}
\hline SEXO & BIMAXILAR & PARCIAL INFERIOR & PARCIAL SUPERIOR & $\begin{array}{c}\text { TOTAL } \\
\text { GENERAL }\end{array}$ \\
\hline $\mathbf{F}$ & $83 \%$ & $10 \%$ & $7 \%$ & 58 \\
$\mathbf{M}$ & $93 \%$ & $5 \%$ & $2 \%$ & 42 \\
Total general & $\mathbf{8 7} \%$ & $\mathbf{8} \%$ & $\mathbf{5 \%}$ & $\mathbf{1 0 0}$ \\
\hline
\end{tabular}

Fig. 1. Frecuencia del Edentulismo por el tipo de dentición según el sexo en los pacientes adultos de la Clínica Integral de la Carrera de Odontología en la Unidad Académica de salud y Bienestar 2016.

\begin{tabular}{lrrrr}
\hline \multicolumn{1}{c}{ EDAD } & BIMAXILAR & $\begin{array}{c}\text { PARCIAL } \\
\text { INFERIOR }\end{array}$ & $\begin{array}{c}\text { PARCIAL } \\
\text { SUPERIOR }\end{array}$ & TOTAL GENERAL \\
\hline DE 51 A MÁS & $92 \%$ & $8 \%$ & 0 & 24 \\
ENTRE 31 Y 50 AÑ̃S & $91 \%$ & $4 \%$ & $6 \%$ & 54 \\
MENOR A 30 & $73 \%$ & $18 \%$ & $9 \%$ & 22 \\
Total general & $\mathbf{8 7}$ & $\mathbf{8}$ & $\mathbf{5}$ & $\mathbf{1 0 0}$ \\
\hline
\end{tabular}

Fig. 2. Frecuencia del edentulismo por el tipo de dentición según la edad. en los pacientes adultos de la Clínica Integral de la Carrera de Odontología en la Unidad Académica de salud y Bienestar 2016.

\section{DISCUSIÓN}

Al realizar la investigación en 100 pacientes adultos de la Clínica Integral de la Carrera de Odontología en la Unidad Académica de salud y Bienestar 2016 se da conocer 


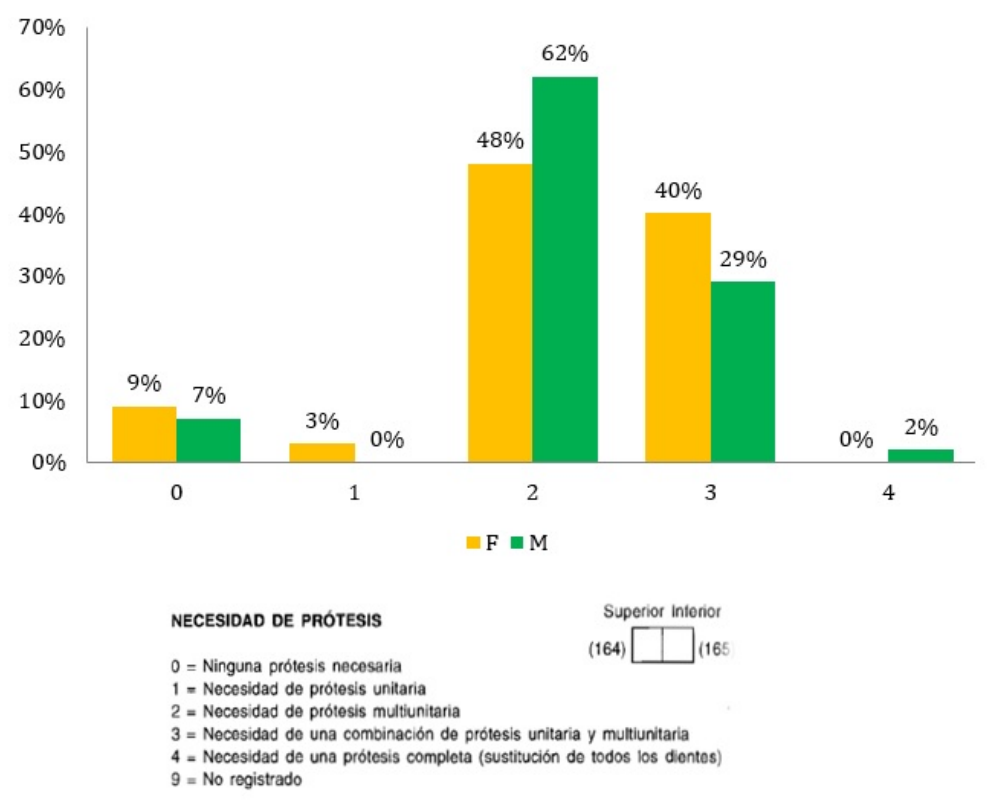

Fig. 3. Frecuencia de la necesidad de tratamiento protésico superior según el sexo en los pacientes adultos de la Clínica Integral de la Carrera de Odontología en la Unidad Académica de salud y Bienestar 2016.

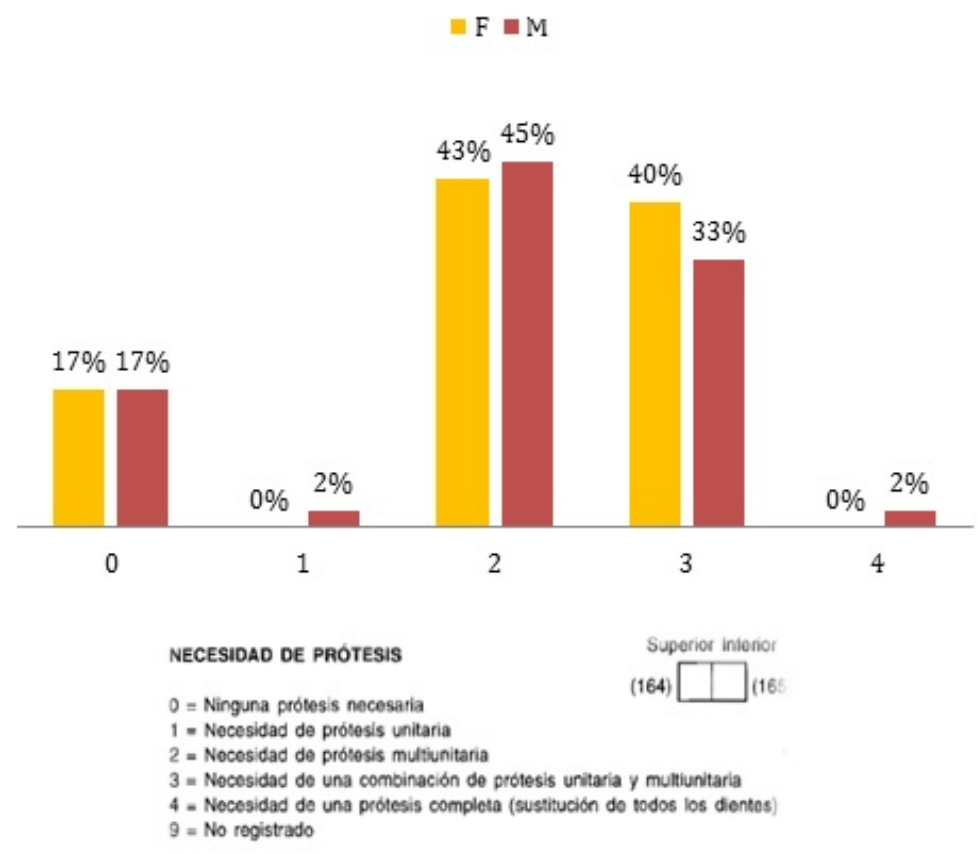

Fig. 4. Frecuencia de la necesidad de tratamiento protésico inferior según el sexo en los pacientes adultos de la Clínica Integral de la Carrera de Odontología en la Unidad Académica de salud y Bienestar 2016. 
el edentulismo y la necesidad de tratamiento protésico.

Se investigó la frecuencia de edentulismo que representa el $100 \%$ de los pacientes estudiados de la Clínica Integral que son Edentulos Parciales bimaxilares con el $87 \%$ y edentulismo parcial superior e inferior con el $13 \%$. Predominando así la necesidad de tratamiento protésico con un porcentaje de $88 \%$, de manera que el $87 \%$ representa la necesidad de tratamiento parcial, el $1 \%$ necesidad de tratamiento total, y el $13 \%$ no requieren tratamiento protésico.

En cambio Vanessa Gutiérrez y cols, ${ }^{11,12}$ en el Golfo de Ventanilla, Lima en el 2014, en su estudio realizado a $121(72 \%)$ personas, donde se clasificó el requerimiento de un tratamiento protésico parcial y total en la arcada superior representando el $(59,6 \%)$ y en la arcada inferior implicando el $(67,3 \%)$. Por lo tanto el edentulismo y el requerimiento de un tratamiento protésico se ve alcanzado en una gran parte de la población. ${ }^{13,14}$

En la presente investigación según la edad nos da a conocer que los más afectados en la necesidad de tratamiento protésico son de edades comprendidas entre 31 a 50 años con $35 \%$ predominando el sexo femenino representando un $(58 \%) .{ }^{15}$

En cambio Osdany Cancio, 2009 ${ }^{16-19}$ investiga la necesidad de prótesis tanto en la edad como en el sexo, revelando que los 355 pacientes entre los 15 a 34 años son los más afectados, representando el $55,7 \%$ y según el sexo el más afectado fue el sexo masculino representando $332(52,1 \%)$ pacientes, en cambio el sexo femenino tuvo un porcentaje de $47.7 \%$ representando los 304 pacientes involucrados de manera que se percibió una mínima diferencia. $^{20,21}$

En la presente investigación la prótesis más necesitada es la parcial con un $87 \%$ la cual es similar a la investigación de Osdany Cancio de manera que la prótesis mas requerida fue la de tipo Parcial con un porcentaje de $83.7 \%$, el $44.9 \%$ representa el sector inferior. En cambio la mayor necesidad de la arcada superior fue la prótesis total representando un $12.3 \%$ que equivale a 127 individuos dando una mínima diferencia entre ambas arcadas. Según el sexo se observa que el mayor requerimiento de prótesis parcial es en el sexo femenino con $44,7 \%$ representando a 461 individuos y en el sexo masculino 402 individuos representando un $38.9 \%$.

En cambio en el requerimiento de prótesis totales se halló 11 en el sexo masculino $149(14.4 \%)$ individuos y en el sexo femenino se halló 19 individuos con $1.8 \%$.

Se analizó según la clasificación de Kennedy en los edentulos parciales, ${ }^{22,23}$ con mayor frecuencia de la clase III en la arcada superior con $29 \%$ de 251 individuos y en el maxilar inferior $200(23,2 \%)$ pacientes.

La clase IV no tuvo mucha representación teniendo solo el $1.8 \%$ equivalente a 16 pacientes de la arcada superior.
Conflicto de intereses y financiamiento Los autores declaran no tener conflicto de intereses, haber cumplido con los requisitos de autoría y haber autofinanciado este artículo.

\section{Referencias}

1 Haydee R, et al. Salud Bucal y Nivel de información en los adultos mayores. Área Norte. Sacti Spíritus.2011. Gac Méd Espirit vol.17 no.3 Sancti Spíritus dic. 2015. Documento disponible

2 Vanessa G, Roberto L. Diana C. Edentulismo y necesidad de tratamiento Protésico en adultos de ámbito urbano marginal. Rev Estomatol Herediana. 2015 Jul-Set; 25(3):179-86. Documento disponible

3 Marro F, et al. Aspectos sobre la medición del impacto de la caries dental en la calidad de vida de las personas. Rev. Clin. Periodoncia Implantol. Rehabil. Oral vol.6 no.1 Santiago abr. 2013 Documento disponible

4 Cancio O. et al. Necesidad de prótesis en pacientes mayores de 15 años. AMC v.13 n.4 Camagüey jul.-ago. 2009. Documento disponible

5 Cristina P, et al. Estado de salud bucal en pobladores del municipio de Palma Soriano. Medisan. 2015.20(5):691. Documento disponible

6 Henriques S, et al. Impacto del Edentulismo en la Calidad de vida de individuos Brasileños. Act. Odont. Venezolana. 2013. Volumen 51 N.3. Documento disponible

7 Sérgio N, Paulo F. et al. Dental health in Brazilian adults between 1986 and 2010. Rev Saúde Pública 2013; 47 (Supl 3):1-9. Documento disponible

8 Galaktion M. Evaluation of edentulism, influence of socioeconomic, behavioural factors and general health on prosthetic status of adult population of georgia. European Scientific Journal. December 2015 /SPECIAL/ edition Vol.2.

9 Medeiros J, Rodrigues L. et al. Edentulismo, Uso e Necessidade de Prótese e Fatores Associados em Município do Nordeste Brasileiro. Pesq Bras Odontoped Clin Integr, João Pessoa. 2012. 1 2(4):573-78.

10 Cunha M, Santos E. et al. Oral Health, Literacy and Quality of Life in the Elderly - Systematic Literature Review. Revista de Enfermagem Referência. Série IV - n 1 - Fev./Mar. 2014.

11 Bellamy $\mathrm{Cl}$, Moreno A. Relación entre calidad de vida relacionada con la salud oral, pérdida dental y prótesis removible en adultos mayores de 50 años derechohabientes del IMSS. Avances en Odontoestomatología Vol. 30. Núm 4. 2014.

12 Sánchez M, Román M. et al. Salud bucal en pacientes adultos mayores y su asociación con la calidad de vida. Revista de Especialidades Médico-Quirúrgicas 2011;16 (2):110115 . 
13 Esquivel R. Jiménez J. Efecto de la utilización de prótesis dentales en la percepción de salud bucal. Revista Adm. Marzo-Abril 2012. Vol. Lxix No. 2.pag. 69-75.

14 Joya LD, Quintero LE. Caracterización de la salud bucal de las personas mayores de 65 años de la ciudad de Manizales, 2008. Hacia promoc. salud. 2015; 20(1): 140-152.

15 Severo da Cruz D, Frazão P. et al. Estudo prospectivo da perda dentária em uma coorte de idosos dentados. Cad. Saúde Pública, Rio de Janeiro, 32(8):e00017215, ago, 2016.

16 PeresI MA, Barbato PR. Perdas dentárias no Brasil: análise da Pesquisa Nacional de Saúde Bucal 2010.Rev Saúde Pública 2013;47(Supl 3):78-89

17 Velásquez G. Prevalencia de la pérdida dentaria en pacientes con osteoporosis.Odous cientifica. Vol 10 N2 Enero- Junio 2009.

18 Aráujo AA. Piuvezam G. e the toothache and toothloss: social representarion of oral care. Ciência \& Saúde Coletiva,11(1):211-218,2006.

19 Agudelo AA, López F. et al. Salud bucal y género en relación a la población adulta mayor atendida en la red hospitalaria pública de Medellín: Los puntos de vista del personal de salud. Rev Univ. salud. 2016;18(1):58-68.

20 Maciel AC, Campos ML. Edentulismo, uso de prótese e autopercepção de saúde bucal entre idosos. Rev Odontol UNESP. 2015 Mar- Apr: 44(2): 74-19

21 Von Marttens A, Carvajal JC. et al. Experiencia y Significado del Proceso de Edentulismo de Adultos Mayores, Atendidos en un Consultorio del Servicio Público Chileno. Rev. Clin. Periodoncia Implantol. Rehábil. Oral. 2010.Vol. 3(1); 27-33.

22 Akinboboye B. et al. Partial Edentulism and unmet prosthetic needs amongst Young adult Nigeria. OdontoStomatologie Tropicale. March 1014. Vol. 37, N 145.

23 Moya P, Chappuzeau E. et al. Situación de salud oral y calidad de vida de los adultos mayores. Rev Estomatol Herediana. 2012; oct-dic 22(4):197-202.

Recibido: 05 de Agosto de 2016

Aceptado: 10 de Diciembre de 2016 
